\title{
Short-term versus long-term mentalization- based therapy for outpatients with subthreshold or diagnosed borderline personality disorder: a protocol for a randomized clinical trial
}

Sophie Juul ${ }^{1,2^{*}} \mathbb{D}$, Susanne Lunn ${ }^{2}$, Stig Poulsen ${ }^{2}$, Per Sørensen ${ }^{1}$, Mehrak Salimi ${ }^{1}$, Janus Christian Jakobsen ${ }^{3}$, Anthony Bateman ${ }^{4}$ and Sebastian Simonsen ${ }^{1}$

\begin{abstract}
Background: Psychotherapy for borderline personality disorder is often lengthy and resource-intensive. However, the current length of outpatient treatments is arbitrary and based on trials that never tested if the treatment intensity could be reduced. As a result, there is insufficient evidence to inform the decision between short-term and long-term psychotherapy for borderline personality disorder. Mentalization-based therapy is one treatment option for borderline personality disorder and consists traditionally of an 18-month treatment program.

Methods/design: This trial is an investigator-initiated single-center randomized clinical superiority trial of short-term (20 weeks) compared to long-term (14 months) mentalization-based therapy for outpatients with subthreshold or diagnosed borderline personality disorder. Participants will be recruited from the Outpatient Clinic for Personality Disorders at Stolpegaard Psychotherapy Centre, Mental Health Services, Capital Region of Denmark. Participants will be included if they meet a minimum of four DSM-V criteria for borderline personality disorder. Participants will be assessed before randomization, and at 8, 16, and 24 months after randomization. The primary outcome is severity of borderline symptomatology assessed with the Zanarini Rating Scale for borderline personality disorder. Secondary outcomes include self-harm incidents, functional impairment (Work and Social Adjustment Scale, Global Assessment of Functioning) and quality of life (Short-Form Health Survey 36). Severity of psychiatric symptoms (Symptom Checklist 90-R) will be included as an exploratory outcome. Measures of personality functioning, attachment, borderline symptoms, group alliance, and mentalization skills will be included to explore potential predictors and mechanisms of change.
\end{abstract}

Discussion: This trial will provide evidence of the beneficial and harmful effects of short-term compared to longterm mentalization-based therapy for outpatients with subthreshold or diagnosed borderline personality disorder.

Trial registration: ClinicalTrials.gov, NCT03677037. Registered on September 19, 2018.

Keywords: Mentalization-based therapy, Borderline personality disorder, Randomized clinical trial, Treatment intensity

\footnotetext{
* Correspondence: sophie.juul@regionh.dk

'Stolpegaard Psychotherapy Centre, Mental Health Services, Gentofte, Capital

Region of Denmark, Denmark

2Department of Psychology, University of Copenhagen, Copenhagen,

Denmark

Full list of author information is available at the end of the article
}

(c) The Author(s). 2019 Open Access This article is distributed under the terms of the Creative Commons Attribution 4.0 International License (http://creativecommons.org/licenses/by/4.0/), which permits unrestricted use, distribution, and

reproduction in any medium, provided you give appropriate credit to the original author(s) and the source, provide a link to the Creative Commons license, and indicate if changes were made. The Creative Commons Public Domain Dedication waiver (http://creativecommons.org/publicdomain/zero/1.0/) applies to the data made available in this article, unless otherwise stated. 


\section{Background}

Borderline personality disorder is a psychiatric condition characterized by a pervasive pattern of symptoms such as interpersonal conflicts, identity diffusion, impulsivity, and emotional dysregulation [1]. According to epidemiological studies, $1.6 \%$ of the general population suffer from borderline personality disorder [2]. In clinical populations, it is the most common personality disorder [2], with a prevalence of between $9 \%$ and $22 \%$ of all psychiatric outpatients [3-5]. Borderline personality disorder is associated with high levels of psychiatric comorbidity, particularly depression, anxiety disorders, eating disorders, substance abuse [6-8], and other personality disorders [9]. Together, these findings emphasize the need for the development of efficacious and cost-effective treatments for this severe and highly prevalent disorder.

While pharmacological treatment may reduce some borderline-related symptoms, there is still no convincing evidence that it is suitable for treating all diagnostic criteria [10]. Although further evidence is still warranted, psychotherapy continues to be the primary treatment of choice for borderline personality disorder [11]. During the last $10-15$ years, studies have established the efficacy of different forms of intensive, specialized long-term psychotherapy modalities. These have recently been evaluated in a systematic review and meta-analysis exploring the efficacy of psychotherapies for borderline personality disorder, in which it was concluded that dialectical behavior therapy and psychodynamic therapies (transference focused therapy and mentalization-based therapy) significantly improved borderline-relevant outcomes [12]. However, no single treatment modality has been established as the primary treatment of choice.

Mentalization-based therapy (MBT) is a psychodynamic therapy rooted in attachment and cognitive theory [13], which was developed specifically for treating borderline personality disorder [14]. Mentalization refers to the capacity to understand one's own and others' mental states. The theoretical assumption is that patients with borderline personality disorder are more vulnerable to lose this capacity when experiencing emotional distress. The MBT manual offers therapeutic techniques to identify these shifts and to bring the patient back into a mentalizing mode [14, 15]. The therapy program consists of four basic components: (1) psycho-education, (2) case formulation, (3) group therapy, and (4) individual therapy. All of these aim to enhance the patient's capacity to mentalize. Increasing mentalization skills is assumed to minimize borderline-related symptoms such as emotional dysregulation, impulsivity, and suicidal ideation. However, information about the mechanisms that produce a change in MBT, or in psychotherapy in general, is still limited [16, 17].
MBT for adult borderline personality disorder has been tested in cohort studies $[18,19]$ and one randomized but uncontrolled trial [20]. Two forms of MBT have been tested in randomized controlled trials: day hospital MBT [21, 22] and intensive outpatient MBT [13], each lasting a maximum of 18 months. For a systematic review of the current evidence base of MBT for borderline personality disorder, see Vogt and Norman [16].

Bateman and Fonagy [13] assessed the effects of intensive outpatient MBT in a randomized clinical trial, in which 134 participants with a confirmed borderline personality disorder diagnosis were randomized either to 18 months of outpatient MBT, combining weekly group and individual sessions with different therapists, or to structured clinical management. In this trial, MBT was superior to structured clinical management in terms of its effects on suicide attempts, severe incidents of self-harm, and on self-reported measures. Treatment effects were sustained at the 5-year follow-up [23]. Nevertheless, only 134 participants were randomized, which questions whether the trial was powered to assess the chosen outcomes, and only 41 were assessed after 5 years. Further, the trial investigators were also the developers of MBT. Thus, the small sample size and the substantial problems with incomplete outcome data, especially at the long-term follow-up, are threats to the validity of the study.

However, while intensive outpatient MBT currently has empirical support as an 18-month program for borderline personality disorder, evidence that this is the optimal length of the intervention is not available. Consequently, MBT is now offered for different lengths of time (both shorter and longer) in outpatient settings around the world [14]. Various other short-term psychotherapies for borderline personality disorder have already been developed and tested in randomized clinical trials, e.g., emotion regulation group therapy [24], systems training for emotional predictability and problem-solving [25, 26], and brief dialectical behavior therapy skills training [27]. However, all the trials have either compared a short-term experimental group to a short-term control group or tested the short-term treatment as an adjunctive to treatment as usual. Thus, these trials do not provide guidance on evidence-based decisions regarding the optimal length of treatment for borderline patients. In addition, no empirical evidence is available to identify which subtypes of patients would benefit from short-term treatment and which would require more intensive treatment [28].

We performed a preliminary literature search (PubMed and Cochrane Library) for trials comparing different lengths of psychotherapy for borderline personality disorder. No such trials were found. When we expanded our search terms to all types of psychiatric disorders, only few trials were identified $[29,30]$. We are 
currently working on a protocol for a more comprehensive systematic review, including a full assessment of risk of bias and a trial sequential analysis of short-term compared with long-term psychotherapy for all psychiatric disorders. The systematic review will be submitted for publication before data collection is completed in this trial.

\section{Methods/design}

\section{Objective}

The primary objective of this trial will be to evaluate the beneficial and harmful effects of short-term (20 weeks) MBT compared with long-term (14 months) MBT for adult outpatients with subthreshold or diagnosed borderline personality disorder. We will evaluate the treatments on the primary outcome (borderline symptomatology), secondary outcomes (self-harm incidents, quality of life, and functional impairment, and exploratory outcomes (psychiatric symptoms). Measures of personality functioning, attachment, borderline psychopathology, group cohesion, and mentalization skills will be included as predictor and mediator variables.

\section{Design}

We have designed an investigator-initiated parallel-group single-centre randomized clinical superiority trial of short-term versus long-term MBT for outpatients with subthreshold or diagnosed borderline personality disorder. The Consolidated Standards of Reporting Trials (CONSORT) flow chart for the trial is shown in Fig. 1. [31, 32]. The Standard Protocol Items: Recommendations for Interventional Trials (SPIRIT) participant timeline is given in Fig. 2, and the SPIRIT checklist is given in Additional file 1 [33].

We will consider for participation all patients referred to the trial site. Patients will be included in the trial, if they comply with the eligibility criteria listed in Table 1. There are inclusion and exclusion criteria as part of the procedure for clinical intake at the trial site, and criteria specific to this trial. For a detailed overview of typical

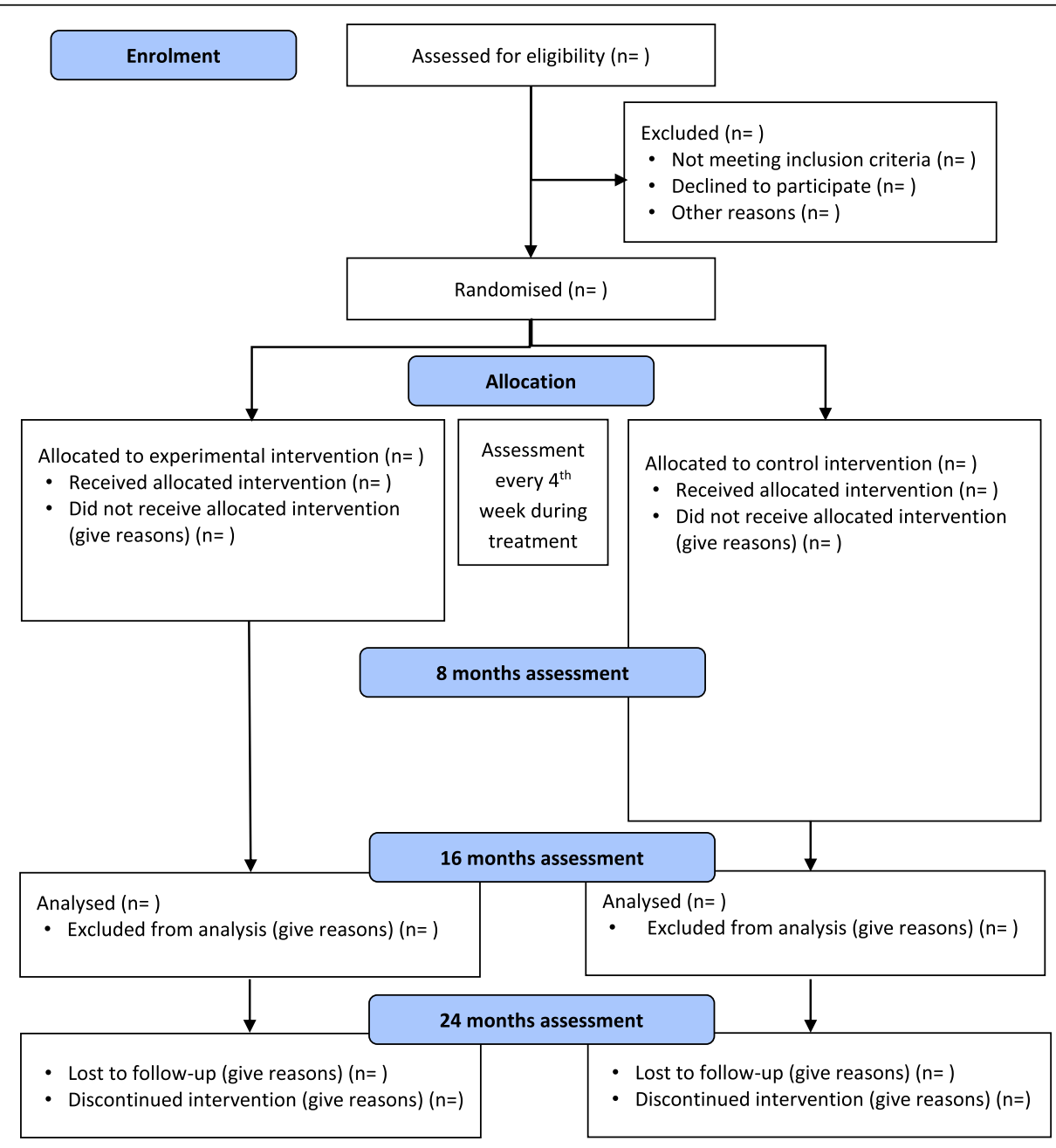

Fig. 1 Consolidated Standards of Reporting Trials (CONSORT) flow chart 


\begin{tabular}{|c|c|c|c|c|c|}
\hline \multirow{3}{*}{ Time point } & \multicolumn{5}{|c|}{ Study period } \\
\hline & \multirow{2}{*}{ Enrolment } & \multirow{2}{*}{ Allocation } & \multicolumn{3}{|c|}{ Post-allocation (months) } \\
\hline & & & 8 & 16 & 24 \\
\hline Enrolment & $-t_{1}$ & 0 & $t_{1}$ & $t_{2}$ & $t_{3}$ \\
\hline Eligibility screening & $\mathrm{x}$ & & & & \\
\hline Informed consent & $\mathrm{x}$ & & & & \\
\hline Allocation & $\mathrm{x}$ & & & & \\
\hline Interventions & & $x$ & & & \\
\hline Short-term mentalization-based therapy & & & & & \\
\hline Long-term mentalization-based therapy & & & & & \\
\hline \multicolumn{6}{|l|}{ Assessments } \\
\hline $\begin{array}{l}\text { Sociodemographic factors, diagnostic } \\
\text { status, personality functioning, } \\
\text { attachment }\end{array}$ & $x$ & & & & \\
\hline $\begin{array}{l}\text { Borderline pathology, self-harm, well- } \\
\text { being, social functioning, psychiatric } \\
\text { symptoms, mentalization skills }\end{array}$ & $x$ & & $x$ & $x$ & $x$ \\
\hline $\begin{array}{l}\text { Group alliance, borderline symptoms, } \\
\text { mentalization skills }\end{array}$ & & & & & \\
\hline
\end{tabular}

Fig. 2 Participant timeline for the Standard Protocol Items: Recommendations for Interventional Trials (SPIRIT)

patient characteristics at the trial site, see Simonsen et al. [34].

We will include participants with at least subthreshold borderline personality disorder. According to the Diagnostic and Statistical Manual of Mental Disorders, 5th edition (DSM-V) [1], the threshold for a full diagnosis is five out of nine diagnostic criteria. However, there is increasing evidence that even four confirmed diagnostic criteria are not qualitatively different from diagnosed borderline personality disorder in terms of impairment, and that the diagnostic threshold should be more inclusive than established by the DSM system to reflect the dimensionality of the construct $[35,36]$. For this reason, previous trials have included participants with at least a subthreshold diagnosis [24,37], and we will do the same in this trial.

\section{Trial site and personnel}

The trial site is the Outpatient Clinic for Personality Disorders at Stolpegaard Psychotherapy Centre, Mental Health Services, Capital Region of Denmark (from now on referred to as "the clinic"). The clinic specializes in MBT for borderline personality disorder. Patients are referred from the Capital Region of Denmark via a central visitation unit, where they are initially screened for eligibility before referral to the clinic. Once referred to the clinic, psychiatrists and attending physicians will perform the initial selection and screening of a participant to the trial and collect informed consent. The principal investigator, sponsor-investigator, or a trained research assistant will then conduct the baseline assessments of the participant. All post-baseline assessments will be carried out by trial investigators who are blind to treatment allocation.

Trial therapists provide therapy to both the short-term and long-term treatment groups. Before commencing the trial, all trial therapists at the clinic will have received training in the short-term MBT program by trial investigators and national and international MBT specialists. The training covers relevant topics like case formulations, termination of psychotherapy, and case-specific supervision. The training will continue throughout the trial period. Therapist treatment fidelity will be rated by an independent certified rater. This will allow us to investigate whether the delivered interventions adhere to the MBT manual.

Table 1 Eligibility criteria

\begin{tabular}{lll}
\hline & Criteria exclusive to the outpatient clinic & Criteria exclusive to the trial \\
\hline $\begin{array}{l}\text { Inclusion } \\
\text { criteria }\end{array}$ & Aged $18-60$ & A minimum of four confirmed DSM-V diagnostic criteria for \\
& Personality disorder(s) considered to be the primary & borderline personality disorder \\
diagnosis/diagnoses & Written informed consent \\
criteria & Possibility of a learning disability $(\mathrm{IQ}<75)$ & Unable to understand Danish \\
& A full diagnosis of schizotypal personality disorder or antisocial & Lack of informed consent \\
& Personality disorder & \\
& Presence of a comorbid psychiatric disorder that requires specialist & \\
& Current (past 2 months) substance dependence including alcohol & \\
& Concurrent psychotherapeutic treatment outside the clinic &
\end{tabular}




\section{Randomization}

Copenhagen Trial Unit, a Danish center for clinical intervention research, will be responsible for the central randomization. Trial investigators will call designated staff at Copenhagen Trial Unit using a central telephone to randomize eligible participants to either the experimental group or the control group with a 1:1 allocation according to a computer-generated allocation sequence with permuted blocks of various sizes generated by Copenhagen Trial Unit and unknown to the investigators, secretaries, and clinical staff at the trial site. This is done to eliminate any predictability in the random sequence. The randomization is stratified by (1) sex and (2) high/low scores on the primary outcome measure, the Zanarini Rating Scale for Borderline Personality Disorder (ZAN-BPD) [38] at baseline.

\section{Interventions}

The short-term MBT program delivered in this trial is overall similar to the existing long-term program, but differs structurally in the following ways: (1) the short-term program is lower in treatment intensity (both duration and exposure), (2) the same therapists provide both group and individual sessions in the short-term program (conjoined psychotherapy), whereas the group therapy and individual therapy are provided by different therapists in the long-term program (combined psychotherapy), and (3) the short-term program is structured in closed groups, in which all participants start and finish the program together, whereas the long-term program is structured as slow-open groups, in which a new participant can enter a group when another finishes. Both interventions in this trial adhere to the treatment guidelines provided by the National Institute for Health and Care Excellence [39].

\section{Experimental intervention}

The short-term MBT program is designed as a 20-week psychotherapy program consisting of five sessions of introductory MBT (MBT-I) followed by 15 sessions of group MBT (MBT-G) accompanied by conjoined individual sessions every second week and two psycho-educative meetings with participants and their relatives. Seven to nine participants and two therapists will be included in each short-term MBT group. The groups are closed to enhance cohesion between group participants. A total of 11 short-term MBT groups will be included in this trial.

Originally, MBT-I was a 12-session introductory psycho-educative program covering relevant topics like personality disorders, attachment, and mentalization [40]. The original manual has been modified for our 5-week intervention. A copy of our modified manual is available upon request. After the completion of MBT-I, the same group of participants will move on to MBT-G, which consists of 15 sessions of mentalization-based psychotherapy in groups, as manualized by Bateman and Fonagy [14]. In our short-term MBT program, group sessions will be accompanied by individual psychotherapy every second week with one of the two group therapists. As part of the individual therapy, a case formulation will be prepared and subsequently shared by the participants in the group. The overall purpose of the individual sessions is for the therapist and participant to develop a consensus of the participant's main difficulties and to establish psychotherapeutic focus points in the group therapy. Furthermore, participants and relatives will be invited to two psycho-educative meetings hosted by the therapists at the beginning of the treatment program to enhance the mentalization work at home. The participants in the short-term MBT program will furthermore be offered three individual follow-up sessions after the end of treatment.

\section{Control intervention}

Long-term MBT is organized as a 14-month program and has been implemented at the clinic for the past 7 years. All participants randomized to long-term MBT will initially enter a 6-week MBT-I program manualized by Karterud and Bateman [40] and modified for our 6-week intervention in collaboration with the authors. New MBT-I groups commence every time new participants are recruited and randomized to long-term MBT. A maximum of 12 participants can enter an MBT-I group. When MBT-I finishes, participants will be allocated to one of eight slow-open MBT treatment groups. MBT-G is then organized as 12 months of weekly group therapy sessions, also manualized by Bateman and Fonagy [14]. In the long-term MBT program, group sessions will be accompanied by combined individual MBT sessions every 2 weeks throughout the program. As part of the individual therapy, a case formulation will be developed and subsequently shared with the group by the participant. Furthermore, participants and relatives will also be invited to two psycho-educative meetings hosted by the therapists to enhance the mentalization work at home. When a participant drops out or completes MBT-G, a new participant can start in the group. This procedure continues until the target sample size has been randomized to the long-term MBT program. The participants in the long-term MBT program will also be offered three individual follow-up sessions after the end of treatment.

\section{Concomitant interventions}

Participants who are receiving psychotropic treatment will be allowed to continue their medical treatment while participating in the trial. The medical protocol will 
follow national as well as international medical recommendations for the treatment of borderline personality disorder and comorbid disorders [39, 41]. Psychiatrists or attending physicians at the clinic will assess the need for additional psychotropic treatment and are asked to adhere to the guidelines. All participants, regardless of treatment condition, will be asked about their current medication by trial personnel during trial interviews to allow us to measure any potential differences in the use of psychotropic medication between the groups.

\section{Baseline assessment at trial intake}

Baseline assessments will be carried out prior to randomization by the principal investigator, the sponsor-investigator, and a trained research assistant, all of whom are also clinical psychologists. General psychopathology will be assessed with the Mini International Neuropsychiatric Interview (MINI) [42]. Personality disorders will be assessed with the Structured Clinical Interview for DSM-V Personality Disorders (SCID-5-PD), formerly known as SCID-II [43]. The SCID-5-PD is considered the gold standard for clinician-administered semi-structured interviews designed to assess personality disorders according to DSM-V criteria [44].

\section{Outcomes}

\section{Primary outcome}

The primary outcome is the severity of borderline symptomatology assessed with the ZAN-BPD [38], which is a clinician-administered scale for the assessment of change in borderline psychopathology over time. Each of the nine borderline personality disorder criteria are rated on a 0 to 4 anchored scale reflecting the severity of symptoms. The rating is intended to reflect both the frequency and the severity of borderline psychopathology. The interview provides a total score of borderline psychopathology ranging from 0 to 36 . ZAN-BPD will be assessed by investigators blind to treatment allocation at baseline, and at the 8-, 16-, and 24-month follow-ups. We will video-record interviews to allow an assessment of inter-rater reliability based on the intraclass correlation coefficient. The results will be evaluated using the guidelines provided by Cicchetti [45].

\section{Secondary outcomes}

Functional impairment will be assessed with the Work and Social Adjustment Scale (WSAS) [46, 47]. This self-report scale will be assessed at baseline, and at the 8-, 16-, and 24-month follow-ups. Quality of life will be assessed with the Short-Form Health Survey (SF-36) [48], which consists of a mental and a physical component. We will use the mental component as a secondary outcome and the physical component as an exploratory outcome. This self-report scale will be given at baseline, and at the 8-, 16-, and 24-month follow-ups. Global functioning will be measured with the Global Assessment of Functioning (GAF) split version [49]. GAF will be assessed by investigators blind to treatment allocation at baseline, and at the 8-, 16-, and 24-month follow-ups. Inter-rater reliability will be calculated using the previously mentioned guidelines. Severe self-harm (dichotomous data) will be measured as the proportion of participants with severe self-harm defined as deliberate acts of self-harm resulting in visible tissue damage. Self-harm will be assessed by investigators blind to treatment allocation using the Suicide and Self-harm Inventory (SSHI) (citation) at baseline, and at the 8-, 16-, and 24-month follow-ups.

\section{Exploratory outcomes}

Symptom distress will be measured with the Global Severity Index (GSI) of the Symptom Checklist 90-R (SCL-90-R) [50]. SCL-90-R will be given at baseline, and at the 8-, 16-, and 24-month follow-ups.

\section{Potential predictors and mediators}

Questionnaires are given at baseline and every fourth week throughout the intervention period for both intervention groups to allow us to explore predictors and mechanisms of change. In a separate statistical analysis plan, which will be submitted for publication before data collection is completed in this trial, we will describe how these exploratory analyses will be performed. The following predictors and mediators will be investigated.

Personality functioning will be assessed with the Levels of Personality Functioning Scale, Brief Form (LPFS-BF) [51], which is a newly developed brief 12-item self-report questionnaire assessing levels of personality functioning according to the DSM-V alternative model for personality disorders. Attachment will be assessed with the brief self-report Relationship Questionnaire (RQ), which gives continuous and categorical ratings of the four attachment styles [52]. Borderline symptomatology will be assessed using the Zanarini Rating Scale for Borderline Personality Disorder, Self-Report Version (ZAN-BPD-SRV) [53]. Group alliance will be assessed using the 12-item version of the Group Questionnaire (GQ) [54], which is a brief self-report measure of the three core components of group alliance: alliance to the other participants, alliance to the therapists, and group cohesion as a whole. Mentalization skills will be assessed with the 15-item Mentalization Questionnaire (MZQ) [55].

For an overview of all measures and the corresponding time of assessment, see Table 2 . 
Table 2 Assessments administered at baseline and each follow-up point throughout the trial

\begin{tabular}{|c|c|c|}
\hline Assessment points & Self-report measures & Expert ratings \\
\hline Baseline & LPFS-BF, RQ, SF-36, WSAS, SCL-90-R, MZQ & MINI, SCID-5-PD, ZAN-BPD, GAF, SSHI \\
\hline Every 4 weeks & GQ, MZQ, ZAN-BPD-SRV & - \\
\hline Follow-ups at 8,16 , and 24 months & SF-36, WSAS, SCL-90-R, MZQ & ZAN-BPD, SSHI, GAF \\
\hline
\end{tabular}

\section{Blinding}

Trial participants and therapists will not be blind to treatment allocation. This is due to the difficulties of implementing an efficient blinding procedure in psychotherapy trials. Baseline assessments will be done before allocation of participants, and the outcome assessments will be performed by blinded assessors. Participants will be instructed to withhold information of their allocation group when assessed. The statistical analyses will be conducted by blinded external statisticians from Copenhagen Trial Unit with the intervention groups coded as A and B. The steering committee will write and agree on two abstracts while the blinding is intact; one assuming the experimental intervention group is $\mathrm{A}$ and the control intervention group is $\mathrm{B}$, and the other assuming the opposite. After this, the randomization code will be broken $[56,57]$.

\section{Participant discontinuation and withdrawal}

Participants can withdraw from the trial at any time without giving a reason and without consequences for future treatment at the clinic. To secure data for the trial, a trial investigator will contact the participant and ask what aspects of the trial the participant wishes to withdraw from: (1) the trial intervention or control group, (2) the assessment interviews, or (3) use of already collected data in analyses. If the participant specifies that they wish to withdraw fully and thereby withdraw from all the points above, their data will be deleted and not used in any analysis. The trial investigator will encourage the participant to continue attending the follow-up assessments.

\section{Data management}

The data in this trial will be collected using electronic case report forms developed in the data collection system REDCap. The system has been approved by the Danish Data Protection Agency and fulfills the requirements for data security. Data from the interviews will be entered directly into REDCap on a tablet, and all self-report measures will be collected from REDCap. For a detailed overview of outcome measures and data collection time points, see Table 2 . The only source data are participants' signed consent forms.
Student assistants employed in the research department but not otherwise involved in the trial will make sure that all self-report measures are sent to participants at the right times, and that the data are complete for all participants enrolled in the trial. The REDCap database has an integrated audit trail to document any access to and changes of the data. The validated data will be exported to SAS for further statistical analyses by statisticians from Copenhagen Trial Unit.

\section{Statistical plan and data analysis Sample size}

The sample size was determined by the predicted change in the primary outcome measure, ZAN-BPD. A 3.5-point superiority margin is considered to be the minimal important difference. Consistent with previous trials that have used ZAN-BPD as an outcome measure for a patient group like ours [24,58], we expect a standard deviation of 8 . With power set at $80 \%$ and alpha set at $5 \%$ two-tailed, a sample size of 83 participants is needed in each treatment group, corresponding to a total of 166 participants.

\section{Statistical methods}

All continuous outcomes will be assessed using linear regression and dichotomous outcomes will be analyzed using logistic regression. The analyses will be based on an intention-to-treat population and will primarily be adjusted for the baseline value of the outcome of interest and the stratification variables used in the randomization. We will secondly adjust all analyses for the following design variables: age (18-30 and 30-60) and functional impairment as assessed with the overall baseline GAF score (0-48 and 49-100) [34].

We will use a five-step procedure [59] to assess if the thresholds for statistical and clinical significance are crossed and we will handle missing data according to the procedure suggested by Jakobsen et al. [60]. A detailed statistical analysis plan will be published before the analyses commence, in which we will provide a detailed description of all primary, secondary, and exploratory analyses. All analyses will be performed blinded with the two intervention groups concealed as A and B. 


\section{Interim analyses}

An external data safety monitoring committee will perform interim analyses when $50 \%$ of the data have been collected according to the good clinical practice guidelines of the International Council for Harmonisation of Technical Requirements for Pharmaceuticals for Human Use [61]. It will decide whether the trial should stop or carry on. Early stopping criteria will follow the recommendations of Jakobsen et al. [59].

\section{Discussion}

This trial will provide evidence of the beneficial and harmful effects of short-term compared to long-term MBT for outpatients with subthreshold or diagnosed borderline personality disorder. To the best of our knowledge, short-term MBT has never been tested before. Gaining more information on how different lengths of treatment work for specific subtypes of patients may help to minimize the potential burden from long-term psychotherapy for some, while at the same time it may identify subtypes of patients for whom short-term psychotherapy is contraindicated. This knowledge may enhance the cost-effectiveness of treatment options for borderline personality disorder. Further, this trial may provide information on the potential predictors and mediators of treatment response.

The present trial has several strengths. First, it has a high degree of external validity because of the relatively inclusive eligibility criteria. Second, the methodology is based on CONSORT, and was predefined and described in detail before randomization began, including, e.g., blinding of all possible parties and implementation of a central randomization system both for generating an allocation sequence and for concealing allocation. Third, the implementation of systematic treatment fidelity ratings allows us to investigate treatment fidelity in both groups.

Our trial also has limitations. First, no systematic review of the effects of short-term compared to long-term psychotherapy for psychiatric disorders is currently available. As mentioned earlier, we are currently performing such a review. Second, the long-term MBT intervention, which is 14 months of treatment in this trial, diverges in intensity (both duration and exposure) from the original 18-month program [13]. This is due to the fixed length of the treatment packages, which have been implemented in the Danish mental health care system. Third, we cannot account for any potential confounding variables because of the structural differences between the groups: the short-term MBT program is closed and conjoined, whereas the long-term program is slow-open and combined.

\section{Dissemination policy}

The Danish population will be informed of the trial as well as its final results through national media. The results of the trial will be presented at all outpatient clinics treating borderline personality disorder in the Mental Health Services, Capital Region of Denmark, by the principal investigator or sponsor-investigator. The final and interim results will be presented at national and international conferences. Further, associations for patients and relatives will be informed about the results of the trial and its future implications. The trial results will be written up by the steering committee and will be published in international peer-reviewed journals. The government of Denmark will be informed of the results before a press release is issued but will have no influence on the reporting of results.

\section{Trial status}

The current protocol is version 1, dated 9 October 2018. The first participant was enrolled on 24 September 2018. Recruitment is expected to be completed by September 1 , 2020

\section{Additional file}

Additional file 1: SPIRIT checklist. (DOCX $61 \mathrm{~kb}$ )

\section{Abbreviations}

CONSORT: Consolidated Standards of Reporting Trials; DSM-V: Diagnostic and Statistical Manual of Mental Disorders, 5th edition; GAF: Global Assessment of Functioning; GQ: Group Questionnaire; GSI: Global Severity Index; LPFS-

BF: Levels of Personality Functioning Scale, Brief Form; MBT: Mentalizationbased therapy; MBT-G: Mentalization-based group therapy; MBT-

I: Introduction to Mentalization-Based Therapy; MINI: Mini International Neuropsychiatric Interview; MZQ: Mentalization Questionnaire;

RQ: Relationship Questionnaire; SCID-5-PD: Structured Clinical Interview for DSM-V Personality Disorders; SCL-90-R: Symptom Checklist 90-R; SF-36: ShortForm Health Survey 36; SPIRIT: Standard Protocol Items: Recommendations for Interventional Trials; SSHI: Suicide and Self-Harm Inventory; WSAS: Work and Social Adjustment Scale; ZAN-BPD: Zanarini Rating Scale for Borderline Personality Disorder; ZAN-BPD-SRV: Zanarini Rating Scale for Borderline Personality Disorder, Self-Report Version

\section{Acknowledgements}

We are grateful to the participants and to our colleagues at the Outpatient Clinic for Personality Disorders at Stolpegaard Psychotherapy Centre for their hard work and good cooperation.

\section{Funding \\ The trial is funded by research grants from TrygFonden and from the Mental Health Services Research Foundation, Capital Region of Denmark. Funding applications have undergone anonymous peer review. Neither the funding bodies nor the sponsor will be involved in the collection, analysis, or interpretation of the data, or in writing the manuscripts. The grants will be administered by the head of administration at Stolpegaard Psychotherapy Center. At the end of the trial, the budget will undergo external auditing independently of the trial sponsor and investigators.}

\section{Availability of data and materials}

The trial investigators, the steering committee, and statisticians at the Copenhagen Trial Unit will have access to the data. After the end of the trial, approval for making the final dataset publicly available in a depersonalized format in the Danish Data Archive will be applied for through the Danish Data Protection Agency. 


\section{Trial sponsor}

The sponsor of the trial is the Mental Health Services in the Capital Region of Denmark, Kristineberg 3, 2100 Copenhagen $\varnothing$.

\section{Protocol amendments}

No substantial deviations from the protocol will be implemented without the prior review and approval of the regulatory authorities, except where such may be necessary to eliminate an immediate hazard to the trial participants. In such case, the deviation will be reported to the regulatory authorities as soon as possible. Any deviation, however minor, will be documented and made available in the trial master file in a designated table developed for this purpose.

\section{Authors' contributions}

SS and SJ conceived the initial idea of a randomized controlled trial comparing short-term and long-term MBT for borderline personality disorder, along with MS, the acting chief psychologist at the clinic. PS approved of the idea and provided organizational support. SS and SJ developed the trial design with contributions and supervision from JCJ, SL, SP, PS, and AB. SJ wrote the protocol, which was carefully discussed with SS, JCJ, SL, SP, PS, and $A B$. JCJ designed the plan for the statistical analyses and wrote the sections on sample size and statistical approach. All authors read and approved the final manuscript.

\section{Ethics approval and consent to participate}

There are no ethical concerns regarding this trial. Both the experimental intervention and control intervention follow the treatment guidelines published by the National Institute for Health and Care Excellence and the Danish Health and Medicines Authority. Any participants can, at any time, withdraw their consent without any implications for future treatment at the clinic. Prior to commencing the trial, ethical approval was obtained from the regional research ethics committee (ID number $\mathrm{H}$-18023136) and approval was obtained from the Danish Data Protection Agency (I-suite number 6553). During the trial period, data on adverse events will be collected at four time points and will be reported to the regional research ethics committee.

\section{Competing interests}

The authors declare that they have no competing interests.

\section{Publisher's Note}

Springer Nature remains neutral with regard to jurisdictional claims in published maps and institutional affiliations.

\section{Author details}

'Stolpegaard Psychotherapy Centre, Mental Health Services, Gentofte, Capital Region of Denmark, Denmark. ²Department of Psychology, University of Copenhagen, Copenhagen, Denmark. ${ }^{3}$ Copenhagen Trial Unit, Center for Clinical Intervention Research, Rigshospitalet, Copenhagen University Hospital, Copenhagen, Denmark. ${ }^{4}$ St. Anns Hospital, London, England.

\section{Received: 11 October 2018 Accepted: 19 March 2019}

Published online: 05 April 2019

\section{References}

1. American Psychiatric Association. Diagnostic and statistical manual of mental disorders (DSM-5 ${ }^{\oplus}$ ). Arlington: American Psychiatric Pub; 2013.

2. Togersen J. In: Oldham J, Skodol AE, Bender DS, editors. Prevalence, Sociodemographics, and Functional Impairment, in Textbook of personality disorders. Washington DC: American Psychiatric Publishing; 2014. p. 109-31.

3. Fossati A, et al. Patterns of covariation of DSM-IV personality disorders in a mixed psychiatric sample. Compr Psychiatry. 2000;41(3):206-15.

4. Zimmerman M, Rothschild L, Chelminski I. The prevalence of DSM-IV personality disorders in psychiatric outpatients. Am J Psychiatr. 2005;162(10): 1911-8.

5. Korzekwa MI, et al. Estimating the prevalence of borderline personality disorder in psychiatric outpatients using a two-phase procedure. Compr Psychiatry. 2008;49(4):380-6.

6. Conklin CZ, Westen D. Borderline Personality Disorder in Clinical Practice. Am J Psychiatr. 2005;162:867-75.
7. Zanarini MC, et al. Axis I comorbidity of borderline personality disorder. Am J Psychiatr. 1998;155:1733-9.

8. Trull TJ, et al. Borderline personality disorder and substance use disorders A review and integration. Clin Psychol Rev. 2000;20(2):235-53.

9. Barrachina J, et al. Axis II comorbidity in borderline personality disorder is influenced by sex, age, and clinical severity. Compr Psychiatry. 2011;52(6):725-30.

10. Lieb K, et al. Pharmacotherapy for borderline personality disorder: Cochrane systematic review of randomised trials. Br J Psychiatry. 2010;196(1):4-12.

11. Bateman AW, Gunderson J, Mulder R. Treatment of personality disorder. Lancet. 2015;385(9969):735-43.

12. Cristea IA, et al. Efficacy of Psychotherapies for Borderline Personality Disorder: A Systematic Review and Meta-analysis. JAMA Psychiatry 2017;74(4):319-28

13. Bateman A, Fonagy P. Randomized controlled trial of outpatient mentalization-based treatment versus structured clinical management for borderline personality disorder. Am J Psychiatr. 2009;166(12):1355-64.

14. Bateman A, Fonagy P. Mentalization-based treatment for personality disorders: a practical guide. Oxford: Oxford University Press; 2016.

15. Karterud S. Mentalization-Based Group Therapy (MBT-G): A theoretical, clinical, and research manual. Oxford: OUP Oxford; 2015.

16. Vogt KS, Norman P. Is mentalization-based therapy effective in treating the symptoms of borderline personality disorder? A systematic review. Psychol Psychother. 2018. https://doi.org/10.1111/papt.12194

17. Kazdin AE. Mediators and mechanisms of change in psychotherapy research. Annu Rev Clin Psychol. 2007;3:1-27.

18. Kvarstein $\mathrm{EH}$, et al. Changing from a traditional psychodynamic treatment programme to mentalization-based treatment for patients with borderline personality disorder--does it make a difference? Psychol Psychother. 2015; 88(1):71-86.

19. Bales $D$, et al. Treatment outcome of 18-month, day hospital mentalizationbased treatment (MBT) in patients with severe borderline personality disorder in the netherlands. J Personal Disord. 2012;26(4):568-82.

20. Jorgensen $C R$, et al. Outcome of mentalization-based and supportive psychotherapy in patients with borderline personality disorder: a randomized trial. Acta Psychiatr Scand. 2013;127(4):305-17.

21. Bateman A, Fonagy P. Effectiveness of partial hospitalization in the treatment of borderline personality disorder: a randomized controlled trial. Am J Psychiatr. 1999;156(10):1563-9.

22. Laurenssen EM, Luyten P, Kikkert MJ, Westra D, Peen J, Soons MB, Dekker JJ. Day hospital mentalization-based treatment $\mathrm{v}$. specialist treatment as usual in patients with borderline personality disorder: randomized controlled trial. Psychol Med. 2018:48(15):2522-29

23. Bateman A, Fonagy P. 8-year follow-up of patients treated for borderline personality disorder: mentalization-based treatment versus treatment as usual. Am J Psychiatr. 2008;165(5):631-8.

24. Gratz KL, Tull MT, Levy R. Randomized controlled trial and uncontrolled 9month follow-up of an adjunctive emotion regulation group therapy for deliberate self-harm among women with borderline personality disorder. Psychol Med. 2014;44(10):2099-112.

25. Blum N, St. John D, Pfohl B, Stuart S, McCormick B, Allen J, Arndt S, Black DW. Systems Training for Emotional Predictability and Problem Solving (STEPPS) for Outpatients With Borderline Personality Disorder: A Randomized Controlled Trial and 1-Year Follow-Up. Am J Psychiatr. 2008;165:468-78.

26. Bos EH, van Wel EB, Appelo MT, Verbraak MJ. A randomized controlled trial of a Dutch version of systems training for emotional predictability and problem solving for borderline personality disorder. J Nerv Ment Dis. 2010; 198(4):299-304

27. McMain SF, et al. A randomized trial of brief dialectical behaviour therapy skills training in suicidal patients suffering from borderline disorder. Acta Psychiatr Scand. 2017;135(2):138-48.

28. McMain SF. Advances in the Treatment of Borderline Personality Disorder: An Introduction to the Special Issue. J Clin Psychol. 2015;71(8):741-6.

29. Knekt $P$, et al. Randomized trial on the effectiveness of long-and shortterm psychodynamic psychotherapy and solution-focused therapy on psychiatric symptoms during a 3-year follow-up. Psychol Med. 2008; 38(5):689-703.

30. Lorentzen $\mathrm{S}$, et al. Comparison of short- and long-term dynamic group psychotherapy: randomised clinical trial. Br J Psychiatry. 2013;203(3):280-7.

31. Boutron I, et al. Extending the CONSORT statement to randomized trials of nonpharmacologic treatment: explanation and elaboration. Ann Intern Med. 2008;148(4):295-309. 
32. Moher D, Schulz KF, Altman DG. The CONSORT statement: revised recommendations for improving the quality of reports of parallel group randomized trials. BMC Med Res Methodol. 2001;1:2.

33. Chan A, et al. SPIRIT 2013 Statement: Defining Standard Protocol Items for Clinical Trials. Ann Intern Med. 2013;158:200-7.

34. Simonsen S, Heinskou T, Sørensen P, Folke S, Lau ME. Personality disorders: patient characteristics and level of outpatient treatment service. Nord J Psychiatry. 2017;71(5):325-31.

35. Clifton A, Pilkonis PA. Evidence for a single latent class of Diagnostic and Statistical Manual of Mental Disorders borderline personality pathology. Compr Psychiatry. 2007;48(1):70-8.

36. Tyrer P, Reed GM, Crawford MJ. Classification, assessment, prevalence, and effect of personality disorder. Lancet. 2015;385(9969):717-26.

37. Beck $E$, et al. Mentalization-based treatment in groups for adolescents with borderline personality disorder (BPD) or subthreshold BPD versus treatment as usual (M-GAB): study protocol for a randomized controlled trial. Trials. 2016:17(1):314

38. Zanarini. Zanarini Rating Scale for Borderline Personality Disorder (ZAN-BPD): a continuous measure of DSM-IV borderline psychopathology. J Personal Disord. 2003;17(3):233-42.

39. National Insitute for Health and Care Excellence (NICE). Borderline Personality Disorder: the NICE Guideline on Treatment and Management. $\mathrm{Br}$ Psychol Soc R Coll Psychiatrists. 2009.

40. Karterud S, Bateman A. Manual for mentaliseringsbasert psykoedukativ gruppeterapi (MBT-I). Oslo: Gyldendal akademisk; 2011.

41. Sundhedsstyrelsen, National klinisk retningslinje for behandling af emotionel ustabil personlighedsstruktur, borderline type. 2015.

42. Lecrubier $Y$, et al. The Mini International Neuropsychiatric Interview (MINI). A short diagnostic structured interview: reliability and validity according to the CIDI. Eur Psychiatry. 1997;12:224-31.

43. First MB, et al. The Structured Clinical Interview for DSM-II-R Personality Disorders (SCID-II). Part II: Multi-site test-retest reliability study. J Personal Disord. 1995;9(2):92-104

44. Lobbestael J, Leurgans M, Arntz A. Inter-rater reliability of the Structured Clinical Interview for DSM-IV Axis I Disorders (SCID I) and Axis II Disorders (SCID II). Clin Psychol Psychother. 2011;18(1):75-9.

45. Cicchetti DV. Guidelines, Criteria, an Rules of Thumb for Evaluating Normed and Standardized Assessment Instruments in Psychology. Psychol Assess. 1994;6(4):284-90

46. Pedersen G, Kvarstein EH, Wilberg T. The Work and Social Adjustment Scale: Psychometric properties and validity among males and females, and outpatients with and without personality disorders. Personal Ment Health. 2017;11(4):215-28.

47. Miles HH, Barrabee EL, Finesinger JE. Evaluation of psychotherapy: with a follow up study of 62 cases of anxiety neurosis. Psychosom Med. 1951 MarApr;13(2):83-105

48. Ware JE, Sherbourne CD. The MOS 36-Item Short-Form Health Survey (SF-36): I Conceptual Framework and Item Selection. Med Care. 1992;30(6):473-83.

49. Jones $\mathrm{SH}$, et al. Reliability and Validity of the Global Assessment of Functioning (GAF). Br J Psychiatry. 1995;166:654-9.

50. Derogatis LR, Cleary PA. Confirmation of the dimensional structure of the SCL-90: a study in construct validation. J Clin Psychol. 1977;33(4):981-9.

51. Hutsebaut J, Feensta D, Kamphuis J. Development and Preliminary Psychometric Evaluation of a Brief Self-Report Questionnaire for the Assessment of the DSM-5 Level of Personality Functioning Scale: The LPFS Brief Form (LPFS-BF). Personal Disord Theory Res Treat 2016;7(2):192-4.

52. Bartholomew K, Horowitz LM. Attachment Styles Among Young Adults: A Test of a Four-Category Model. J Pers Soc Psychol. 1991;61(2):226-44.

53. Zanarini, et al. Development of the self-report version of the Zanarini Rating Scale for Borderline Personality Disorder. Personal Ment Health. 2015;9(4):243-9.

54. Krogel J, et al. The Group Questionnaire: a clinical and empirically derived measure of group relationship. Psychother Res. 2013;23(3):344-54.

55. Hausberg M, Schulz H, Piegler T, Happach C, Klöpper M, Brütt A, et al. Is a self-rated instrument appropriate to assess mentalization in patients with mental disorders? Development and first validation of the Mentalization Questionnaire (MZQ). Psychother Res. 2012;22(6):699-709.

56. Gotzsche PC. Blinding during data analysis and writing of manuscripts. Control Clin Trials. 1996;17(4):285-90 discussion 290-3.

57. Jarvinen $T L$, et al. Blinded interpretation of study results can feasibly and effectively diminish interpretation bias. J Clin Epidemiol. 2014;67(7):769-72.
58. Priebe S, et al. Effectiveness and cost-effectiveness of dialectical behaviour therapy for self-harming patients with personality disorder: a pragmatic randomised controlled trial. Psychother Psychosom. 2012;81(6):356-65.

59. Jakobsen $\mathrm{JC}$, et al. The thresholds for statistical and clinical significance - a five-step procedure for evaluation of intervention effects in randomised clinical trials. BMC Med Res Methodol. 2014;14:34.

60. Jakobsen JC, et al. When and how should multiple imputation be used for handling missing data in randomised clinical trials - a practical guide with flowcharts. BMC Med Res Methodol. 2017;17(1):162.

61. Englev E, Petersen KP. ICH-GCP Guideline: quality assurance of clinical trials. Status and perspectives. Dan Med J. 2003;165(16):1659-62.
Ready to submit your research? Choose BMC and benefit from:

- fast, convenient online submission

- thorough peer review by experienced researchers in your field

- rapid publication on acceptance

- support for research data, including large and complex data types

- gold Open Access which fosters wider collaboration and increased citations

- maximum visibility for your research: over $100 \mathrm{M}$ website views per year

At $\mathrm{BMC}$, research is always in progress.

Learn more biomedcentral.com/submissions 